\title{
Soldabilidad de Polietileno de Alta Densidad Usando Soldadura por Fricción-agitación con una Herramienta de Hombro no Rotacional
}

\author{
Yorledis Macea Romero ${ }^{1}$, Mirley Moreno Moreno ${ }^{1}$, Beatriz Arrieta Cardozo ${ }^{1}$, William Plata Rueda ${ }^{2}$, \\ Salomón Consuegra Pacheco², Jimy Unfried-Silgado \\ ${ }^{1}$ Universidad Autónoma del Caribe, Facultad de ingeniería, Grupo IMTEF, Barranquilla, Colombia. \\ ${ }^{2}$ Institución Universitaria - ITSA, Escuela de Procesos Industriales, Barranquilla, Colombia. \\ ${ }^{3}$ Universidad de la Costa, Ingeniería Industrial, Grupo PRODUCOM, Barranquilla, Colombia.
}

Recibido: 23 Ago., 2016

Aprobado: 02 Fev., 2017

E-mail: junfried1@cuc.edu.co (JUS)
Este es un artículo publicado en acceso (Open Access) abierto bajo la licencia Creative Commons Attribution NonCommercial, que permite su uso, distribución y reproducción en cualquier medio, sin restricciones siempre que sin fines comerciales y que el trabajo original sea debidamente citado.
Resumen: En este trabajo se estudió la soldabilidad de polietileno de alta densidad (PEAD) mediante el proceso de soldadura por fricción agitación (SFA), usando dos herramientas diferentes, una convencional y otra con hombro no rotacional, denominada no convencional. La evaluación comparativa fue realizada bajo combinaciones de los parámetros de velocidad de rotación $\left(\mathrm{v}_{\mathrm{w}}\right)$ y velocidad de avance $\left(\mathrm{v}_{\mathrm{A}}\right)$ ajustados para cada herramienta. La soldabilidad fue valorada con base en inspección visual, la presencia de discontinuidades mediante estereoscopia y microscopia confocal y la dureza en las juntas soldadas. Como resultado se obtuvo que la herramienta no convencional permitió obtener juntas soldadas libres de discontinuidades y con excelente apariencia externa gracias al menor calor aportado. La dureza en la zona agitada fue menor a la del material base, mostrando comportamientos diferenciados en los lados de avance y retroceso en función de los parámetros de proceso.

Palabras-clave: Soldadura por Fricción Agitación (FSW); Polietileno; Discontinuidades; Soldabilidad.

\section{Weldability of High Density Polyethylene Using Friction Stir Welding with a Non-rotational Shoulder Tool}

\begin{abstract}
In this work was studied the weldability of high density polyethylene (HDPE) using friction stir welding (FSW) process, and two kind of tools, denominated conventional, and non-conventional tool, which has a non-rotating shoulder. Evaluation was performed using combination of parameters of rotation speed $\left(v_{w}\right)$ and welding speed $\left(v_{A}\right)$, which were adjusted for each type of tool. Weldability was estimated based on visual inspection, the presence of discontinuities, and tests of stereoscopy and confocal microscopy, and hardness in welded joints. Results shows that the unconventional tool obtains welded joints free of discontinuities and an excellent external appearance due to low heat input. The hardness decreased in the stirred zone compared to material base, showing different behavior on the retreating and advancing sides, depending on the process parameters.
\end{abstract}

Key-words: Friction Stir Welding (FSW); High Density Polyethylene (HDPE); Discontinuities; Weldability.

\section{Introducción}

A pesar de que la mayoría de los polímeros termoplásticos pueden ser moldeados fácilmente hasta obtener la forma general deseada, muchas piezas de estos materiales requieren unión mecánica sin adhesivos para la obtención de la forma final. Por esta razón, en los últimos años ha cobrado gran interés la implementación de soldaduras en estado sólido para materiales termoplásticos [1]. Uno de los procesos más promisorios para este tipo de uniones es la soldadura por fricción-agitación (SFA) (- por su acrónimo en inglés Friction Stir Welding - FSW), teniendo en cuenta que este proceso en metales crea una unión permanente de alta calidad, con alta resistencia y baja distorsión. Con este proceso es posible fabricar juntas soldadas en termoplásticos a tope u orbitales, en una amplia gama de espesores y longitudes [2]. La soldadura por SFA es una de las tecnologías de unión que permite reducir el desperdicio de material, el exceso de emisiones por radiación y/o convección y de gases nocivos normalmente asociados con las técnicas de soldadura por fusión [3]. 
El proceso SFA, en su versión tradicional, utiliza una herramienta rotacional no consumible, cuyo cuerpo en un extremo posee un pin y un hombro solidarios en giro. Esta herramienta presenta dos funciones que son: generar calor localizado debido a la fricción entre esta y la pieza de trabajo y hacer fluir el material, confinándolo (a manera de forjado) y empujándolo en la junta (a manera de extrusión). De esta forma, mientras que la herramienta gira a determinadas revoluciones por minuto entre dos placas fijas $\left(v_{w}\right)$, el pin penetra de forma axial las piezas de trabajo, hasta que el hombro entra en contacto con la cara superior de estas. La continua fricción entre el hombro, el pin y la pieza genera una cantidad de calor que provoca el ablandamiento del material, bajo un determinado tiempo de permanencia (dwell time). Sea la herramienta o la placa, dependiendo del tipo de máquina disponible para el proceso, se le induce una velocidad de avance $\left(v_{A}\right)$, provocando que el material ablandado fluya desde el frente de la herramienta hacia atrás en la trayectoria destinada, para así producir la junta soldada [4]. Los parámetros y partes fundamentales de una junta soldada por SFA se muestran en la Figura 1.

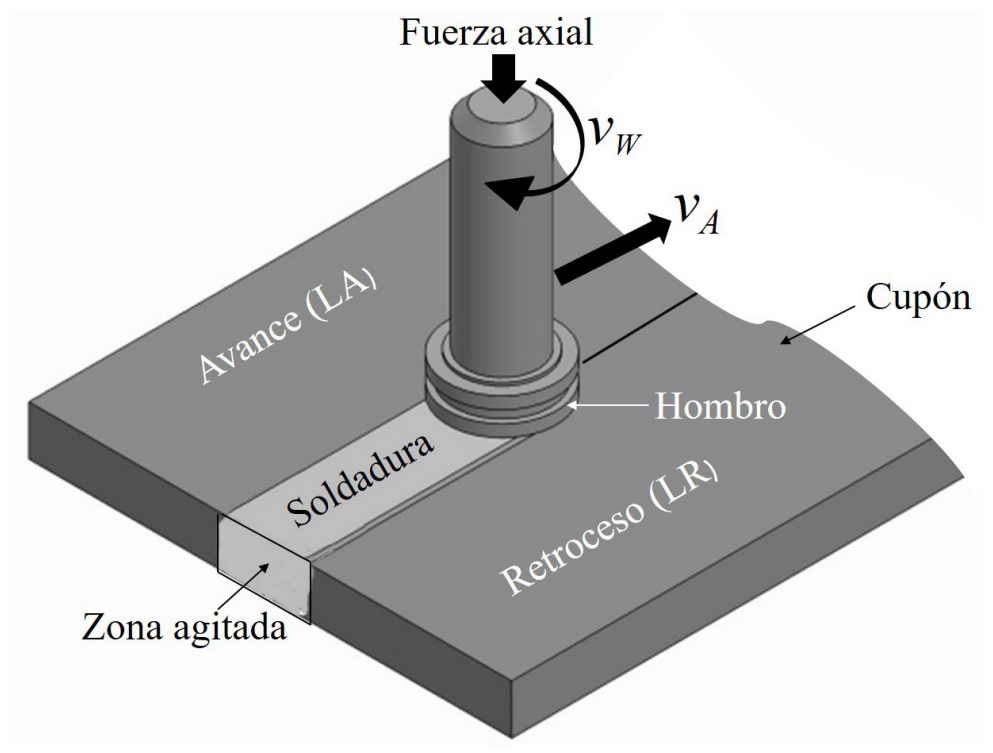

Figura 1. Esquema de partes de una junta y parámetros del proceso de Soldadura por Fricción - Agitación.

El proceso de soldadura por fricción-agitación fue inventado y patentado por W. Thomas en el instituto de soldadura (TWI) en 1991 [5]. Aunque este proceso fue aplicado inicialmente en aleaciones de aluminio y otras aleaciones ligeras, recientemente algunos investigadores han estudiado la aplicación de SFA en termoplásticos. La obtención de juntas con calidad es una tarea compleja en polímeros, debido a que los termoplásticos están constituidos por moléculas de cadena larga de alto peso molecular, en lugar de átomos individuales, las cuales infieren diferentes características de flujo de material durante la soldadura. Por lo tanto, los parámetros del proceso y diseños de herramientas para la SFA de termoplásticos son bastante diferentes de aquellos requeridos para la soldadura de metales [2-4].

Diversos autores han estudiado los efectos de modificaciones de la forma de herramientas en el proceso de SFA aplicado en polímeros termoplásticos. Panneerselvam y Lenin [6], determinaron el efecto en la dirección de rotación en una herramienta con pin cilíndrico roscado sobre las propiedades mecánicas en juntas de Nylon 6 . Estos autores encontraron que, cuando la dirección de flujo difiere de la dirección de rotación de la herramienta (el flujo de material es confinado hacia abajo), las propiedades mecánicas aumentan. Kiss y Czigány [7] estudiaron la estructura molecular de uniones soldadas por SFA a través de microscopia óptica y electrónica de uniones soldadas por SFA, en la cuales, a diferencia de la soldadura en metales se utilizó una zapata estática hecha de politetrafluoroetileno, suavizada y sin calentamiento. Estos autores demostraron que, en la parte central de este tipo de juntas soldadas, las esferulitas formadas son similares a las del material base. De otro lado, en el límite de la junta, se identificó una estructura supramolecular más compleja. Rezgui et al. [8], aplicaron un modelo estadístico de Taguchi para optimizar los parámetros de SFA en polietileno de alta densidad usando juntas a tope. Los parámetros estudiados por estos autores fueron velocidad de avance, velocidad de rotación, diámetro del pin y tiempo de sostenimiento. 
Soldabilidad de Polietileno de Alta Densidad Usando Soldadura por Fricción-agitación con una Herramienta de Hombro no Rotacional

Usaron un diseño de herramienta especial para polímeros cuyo hombro es estático, aislando las muestras soldadas y conservando el calor ganado por la fricción evitando así la aparición de discontinuidades después de la soldadura. Estos autores, al final analizaron las variaciones en la temperatura de la junta durante el proceso y los esfuerzos de tensión, obteniendo resultados coherentes con las predicciones numéricas en cuanto a comportamiento mecánico para diferentes condiciones de soldadura en este material. Por otro lado, Inaniwa et al. [9] seleccionaron distintos materiales termoplásticos, entre ellos, polietileno de alta densidad (PEAD), poliamida 6 (PA6) y policlroruro de vinilo (PVC), con el fin de investigar mediante ensayos de dureza y de tracción el efecto de las diferencias en las propiedades físicas de cada material sobre la condición de soldabilidad y la resistencia mecánica en la unión. Estos autores encontraron que la relación entre la velocidad de avance y la velocidad de rotación, para cada material, se incrementa con el decrecimiento de la viscosidad. Observaron, además que la zona agitada presentó menor dureza comparada al material base, obteniéndose valores de eficiencia de la junta del $70 \%, 45 \%$ y $35 \%$, para PEAD, PVC y PA6, respectivamente. Finalmente, estos autores obtuvieron como parámetros óptimos para soldar PEAD, velocidades de rotación $\left(v_{w}\right)$ entre 800 a $1200 \mathrm{rpm}$ y velocidades de avance $\left(v_{A}\right)$ entre 15 a $45 \mathrm{~mm} \cdot \mathrm{min}^{-1}$. La máxima eficiencia en la junta fue reportada entre 50 a 70\% con relaciones de velocidades entre 0,01 a 0,02 mm.rev ${ }^{-1} \mathrm{con}^{-}$ un esfuerzo máximo en la junta $\sigma_{\mathrm{ut}}$ entre 20 a $25 \mathrm{MPa}$.

El objetivo de este trabajo es evaluar la influencia de los parámetros de proceso, velocidad de rotación $\left(v_{w}\right)$ y velocidad de avance $\left(v_{A}\right)$ sobre la soldabilidad de placas de polietileno de alta densidad industrial usando el proceso de soldadura por fricción-agitación y una herramienta con hombro no rotacional, con el fin de identificar ventanas de proceso que produzcan soldaduras sanas con propiedades homogéneas.

\section{Materiales y Métodos}

\subsection{Materiales, herramientas y equipos}

El material base evaluado consistió en placas comerciales de Polietileno de alta densidad (PEAD), extruido y reprocesado con dimensiones de $61,5 \mathrm{~mm} \times 122 \mathrm{~mm} \times 8,5 \mathrm{~mm}$. La trazabilidad del sentido de extrusión de las láminas ha sido observada durante todo el proceso, de tal manera que los cordones de soldadura han sido diseñados para aplicarse de forma perpendicular al sentido de extrusión. Se diseñaron y elaboraron dos herramientas para soldadura por fricción-agitación en acero AISI H-13, las cuales se muestran en la Figura 2.

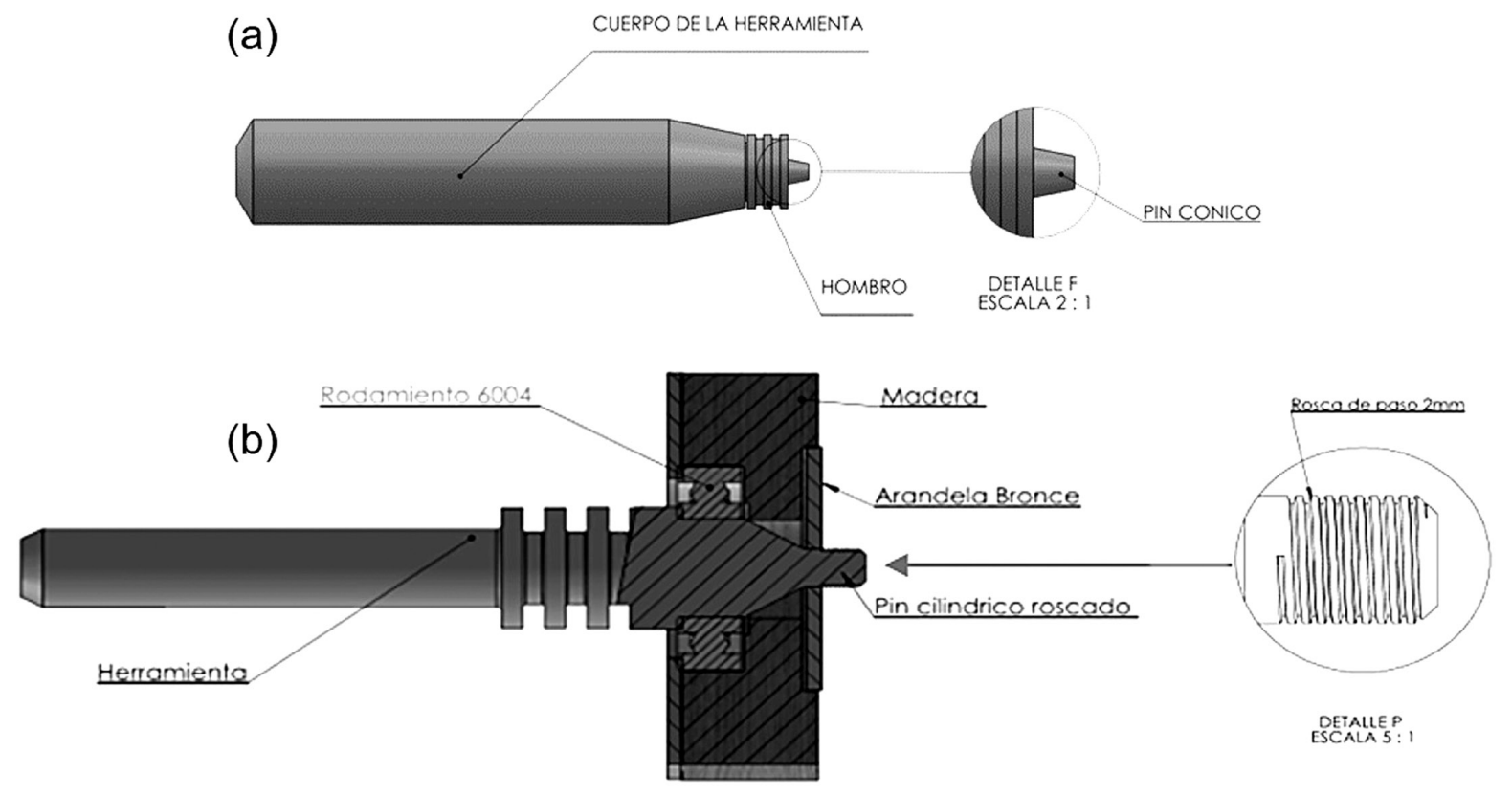

Figura 2. Diseño de las herramientas. a) Convencional y b) Con hombro no rotacional. 
La herramienta mostrada en la Figura 2a es de tipo convencional, con pin cónico roscado y hombro solidario rotacional con el eje. La otra herramienta (Figura $2 \mathrm{~b}$ ) es no convencional, con pin cilíndrico roscado y hombro no rotacional. Para lograr esto último, el cuerpo de la herramienta se sujetó a un bloque monolítico de madera mediante un rodamiento, donde una placa de metal estática no conectada al pin sustituyó al hombro [8]. La dirección de avance y el sentido de rotación para cada herramienta fueron previamente seleccionados.

Para el desarrollo de las soldaduras se usó una máquina fresadora universal convencional Imomill ${ }^{\circledR}$ de $3,2 \mathrm{hp}$ de potencia nominal. En esta fresadora las herramientas fueron correctamente acopladas y se implementó un sistema de sujeción para fijar las placas a soldar, el cual se adaptó a la bancada de la fresadora, ajustándolo a las dimensiones de esta, de tal forma que fuese posible realizar libremente el recorrido de la soldadura para ambas herramientas, como se muestra en la Figura 3.

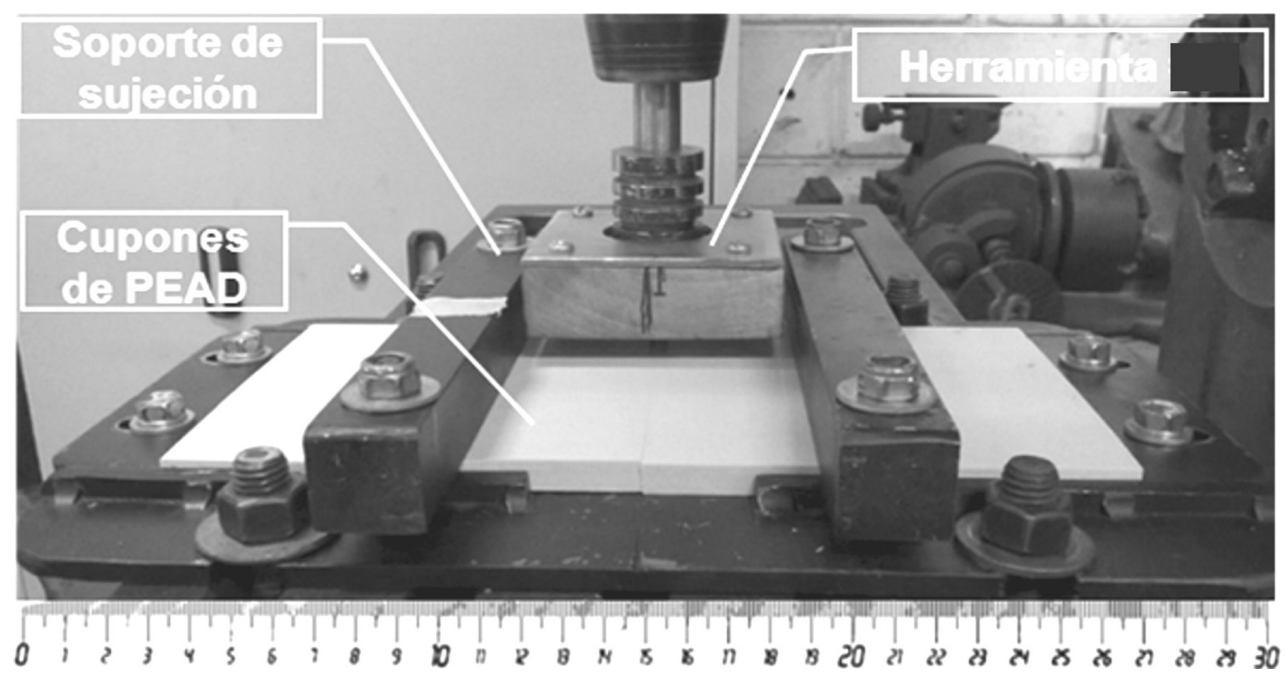

Figura 3. Vista frontal del montaje del proceso de FSW en PEAD.

\subsection{Ventanas de proceso y soldaduras preliminares}

Se realizaron inicialmente soldaduras preliminares para cada uno de los tipos de herramientas, de acuerdo a la configuración mostrada en la Figura 4. Se realizaron las soldaduras de forma perpendicular al sentido de laminación con sentido de rotación de la herramienta antihorario [6].

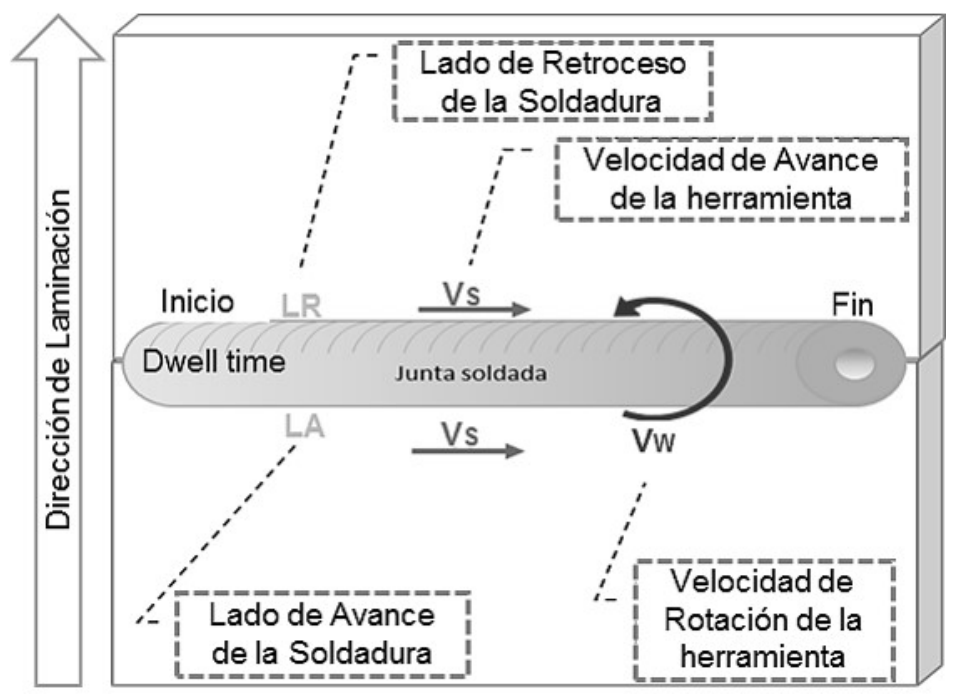

Figura 4. Configuración geométrica de las soldaduras en PEAD. 
Soldabilidad de Polietileno de Alta Densidad Usando Soldadura por Fricción-agitación con una Herramienta de Hombro no Rotacional

Para la determinación de la ventana de procesos se ha utilizado combinaciones de los parámetros mostrados en la Tabla 1, con los cuales se realizaron juntas soldadas con las herramientas convencional y no convencional. Los valores iniciales de los parámetros utilizados experimentalmente fueron extraídos de datos provenientes de la literatura [8-10]. Cada conjunto fue validado mediante juntas soldadas sanas y sin discontinuidades visibles, de acuerdo a los criterios establecidos en la siguiente sección.

Tabla 1. Valores de parámetros de proceso usados.

\begin{tabular}{lcc}
\hline \multicolumn{1}{c}{ Parámetros } & \multicolumn{2}{c}{ Tipo de herramienta } \\
\cline { 2 - 3 } & Convencional & No convencional \\
Velocidad de rotación $-v_{W}(\mathrm{rpm})$ & 250 a 1036 & 846 a 1036 \\
Velocidad de avance $-v_{A}\left(\mathrm{~mm} \cdot \mathrm{min}^{-1}\right)$ & 14 a 36 & 14 a 25 \\
\hline
\end{tabular}

Para cada conjunto de valores de parámetros de proceso se estableció la relación $(R)$ entre velocidad de avance $\left(v_{A}\right)$ y velocidad de rotación $\left(v_{w}\right)$, de acuerdo con la Ecuación 1 , la cual expresa la relación de entrada de calor por cada revolución de la herramienta para cada junta soldada.

$$
R=\frac{v_{A}}{v_{W}} \quad\left[m m \cdot r e v^{-1}\right]
$$

\subsection{Criterios de selección de soldaduras}

Las juntas soldadas (preliminares y finales) se examinaron por inspección visual y se evaluó físicamente la integridad de la junta de acuerdo a los siguientes criterios: rebabas con tamaño (altura) menor a $2 \mathrm{~mm}$, existencia de un cordón continúo con más de $30 \mathrm{~mm}$ de longitud, que no posea defectos del tipo pin-hole (vacío en el interior del cordón), no presentar vacíos, ni discontinuidades en la superficie del cordón y rugosidad uniforme en la junta y sobre el cordón de soldadura.

Se consideraron soldaduras aceptables a todas aquellas juntas soldadas que cumplan los criterios antes mencionados. Usando estos criterios se definieron los valores de parámetros de soldadura que se escogieron como la ventana de procesos a utilizar.

\subsection{Caracterización de soldaduras}

\subsubsection{Cuerpos de prueba de juntas soldadas}

La extracción de cuerpos de prueba de las juntas soldadas se realizó mediante cortes transversales al cordón de soldadura, usando una cuchilla metálica a baja velocidad para evitar calentamiento, como se muestra en la Figura 5. Se extrajeron probetas con dimensiones de $20 \mathrm{~mm} \times 10 \mathrm{~mm} \times 8,5 \mathrm{~mm}$, para cada conjunto de parámetros ensayado, todas ellas provenientes desde la zona central del cordón de soldadura para garantizar distribución de calor estacionaria.

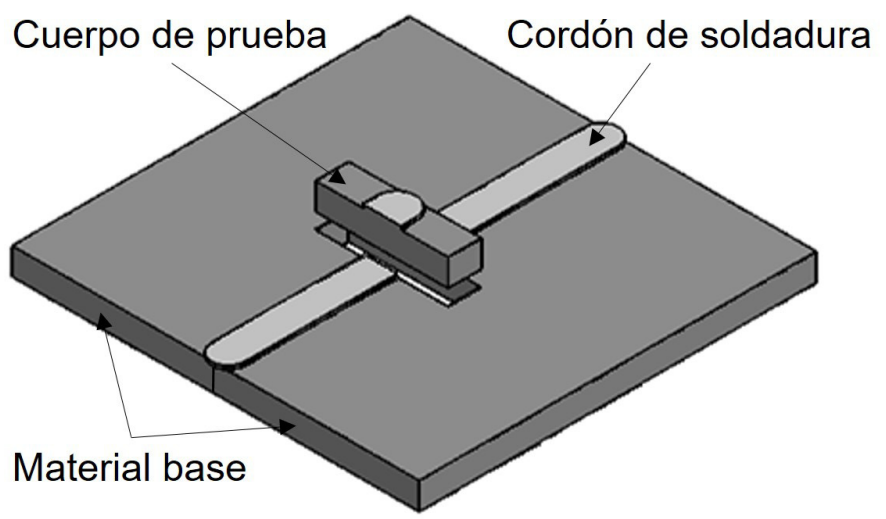

Figura 5. Localización y extracción de los cuerpos de prueba para caracterización. 


\subsubsection{Microscopia confocal láser y estereoscopia óptica}

Con el fin de observar los detalles de las zonas de soldadura y verificar la presencia de posibles discontinuidades se utilizó microscopía confocal láser para la observación de la sección transversal de la junta de soldadura para cada conjunto de parámetros. El equipo usado fue un microscopio Olympus ${ }^{\circledR}$ Lext 30 Measuring Laser Microscope OLS4100. Así mismo, se tomaron macrografías de la sección transversal con un estereoscopio óptico Leica ${ }^{\circledR}$ EZ4 HD. Las probetas se prepararon con lijado suave desde granulometría 300 hasta 1500 de acuerdo a la norma ASTM E2015-04.

\subsubsection{Barridos de dureza}

Se realizaron barridos de dureza sobre las mismas probetas empleadas para microscopia y estereoscopia en secuencias de tres barridos por cada combinación de parámetros con una separación por serie de barridos de 2,6 mm como se muestra en la Figura 6 . Cada barrido consta de 5 identaciones con una separación de $4 \mathrm{~mm}$ durante un tiempo de $1 \mathrm{~s}$. El ensayo se realizó bajo norma ASTM D2240-05 [11] empleando un durómetro comercial Shore Digital Tipo D.

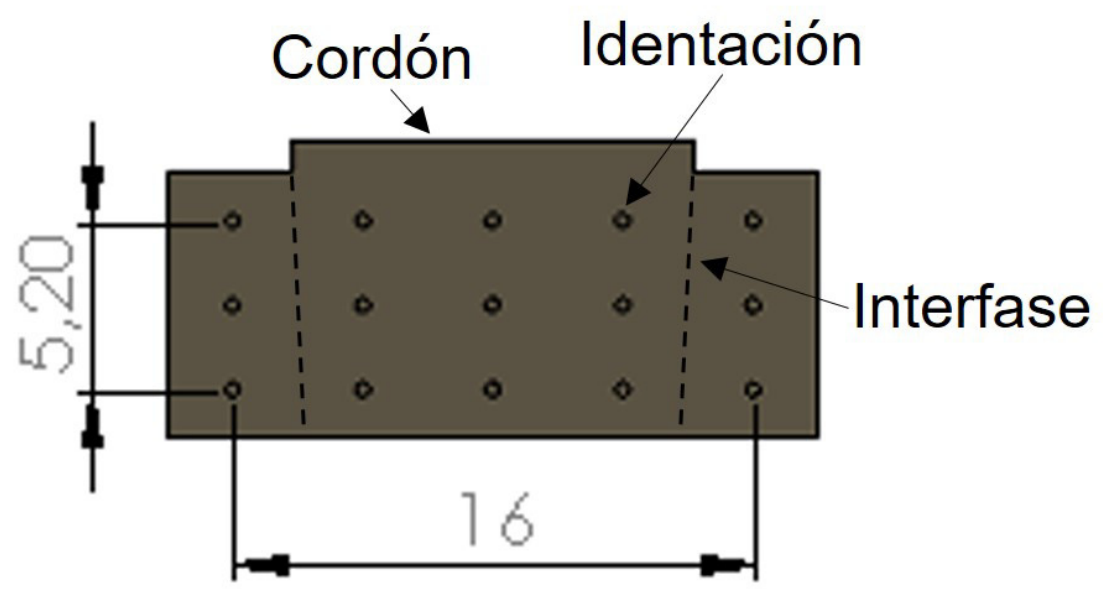

Figura 6. Probetas para ensayos de dureza y ubicación de barridos.

\section{Resultados y Discusión}

\subsection{Soldaduras preliminares con herramienta convencional}

Los resultados de las soldaduras preliminares realizadas con herramienta convencional, usados para la evaluación de la ventana de procesos, son mostrados en la Figura 7. Se puede observar que las juntas presentaron discontinuidades críticas, como vacíos (continuos o discontinuos) y rebabas superiores a $2 \mathrm{~mm}$, que llevan a considerar que estas no superaron los criterios de selección por lo que fueron rechazadas.

Las discontinuidades observadas en las soldaduras obtenidas con la herramienta convencional, principalmente están relacionadas, con el mayor calor por fricción generado gracias a la acción del hombro rotatorio de esta herramienta, dando lugar al surgimiento de rebabas y a fusión parcial, evitando la consolidación de la junta. A medida que la velocidad de avance $\left(v_{A}\right)$ aumenta, también se aumentan los vacíos, falta de caldeo en el material $y$, en algunos casos, incluso no se genera una soldadura. Ninguna de las juntas generó uniones satisfactorias, por lo que se descarta el uso de esta herramienta convencional para soldar PEAD bajo estas condiciones.

\subsection{Soldaduras preliminares con herramienta no convencional}

Los resultados de algunas de las soldaduras preliminares realizadas con herramienta no convencional, usados para la evaluación de la ventana de procesos, son mostrados en la Figura 8. Se evidenció que las soldaduras hechas con esta herramienta no convencional, la cual no posee movimiento rotacional del hombro, da lugar a soldaduras continuas y con baja cantidad de defectos visuales en comparación con las soldaduras obtenidas con la herramienta convencional bajo condiciones de proceso similares. 
Soldabilidad de Polietileno de Alta Densidad Usando Soldadura por Fricción-agitación con una Herramienta de Hombro no Rotacional
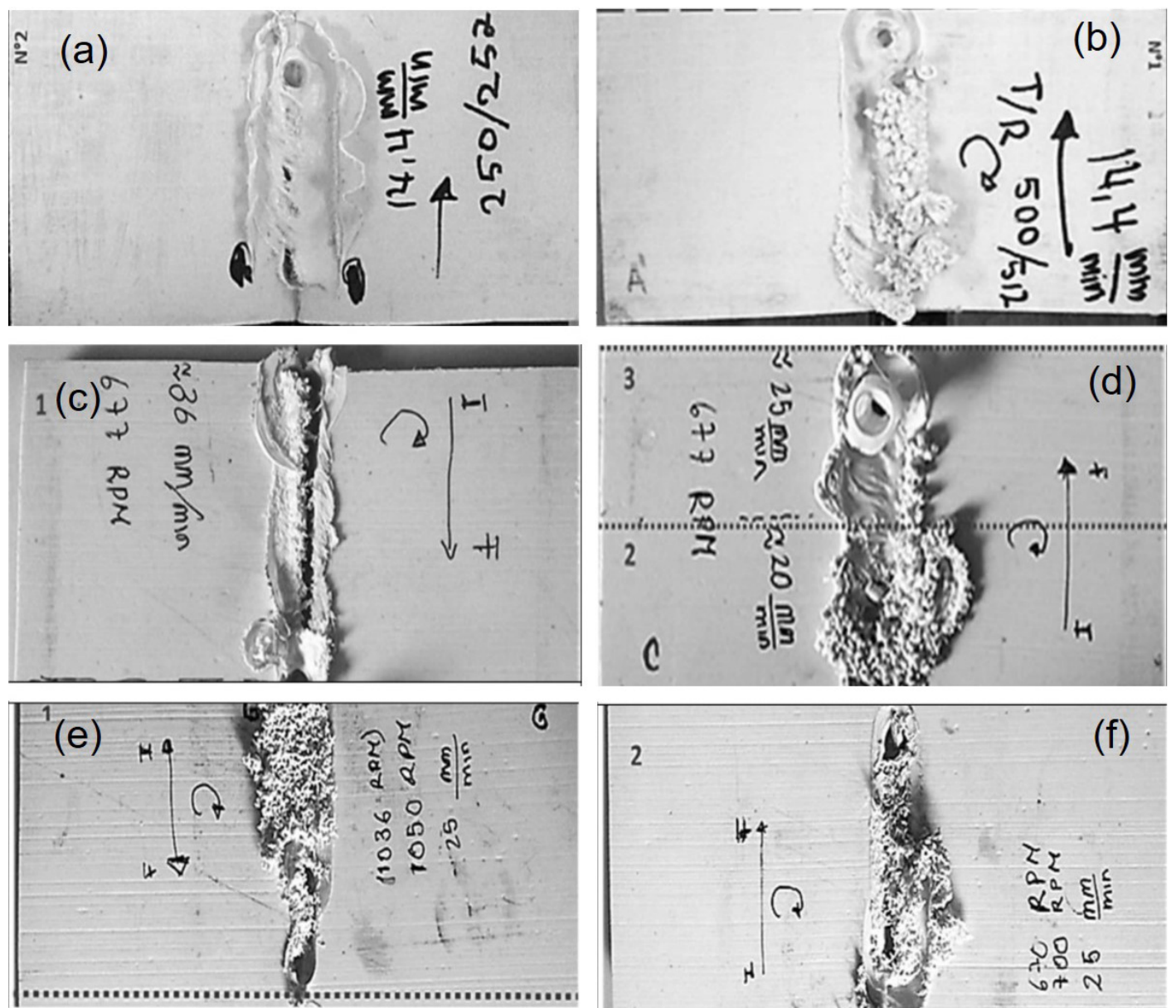

Figura 7. Soldaduras preliminares con herramienta convencional. (a) $252 \mathrm{rpm} / 14 \mathrm{~mm} \cdot \mathrm{min}^{-1}$; (b) $512 \mathrm{rpm} / 14 \mathrm{~mm} \cdot \mathrm{min}^{-1}$; (c) $677 \mathrm{rpm} / 36 \mathrm{~mm} \cdot \mathrm{min}^{-1}$; (d) $677 \mathrm{rpm} / 20 \mathrm{~mm} \cdot \mathrm{min}^{-1}$; (e) $1036 \mathrm{rpm} / 25 \mathrm{~mm} \cdot \mathrm{min}^{-1}$; (f) $670 \mathrm{rpm} / 25 \mathrm{~mm} \cdot \mathrm{min}^{-1}$.
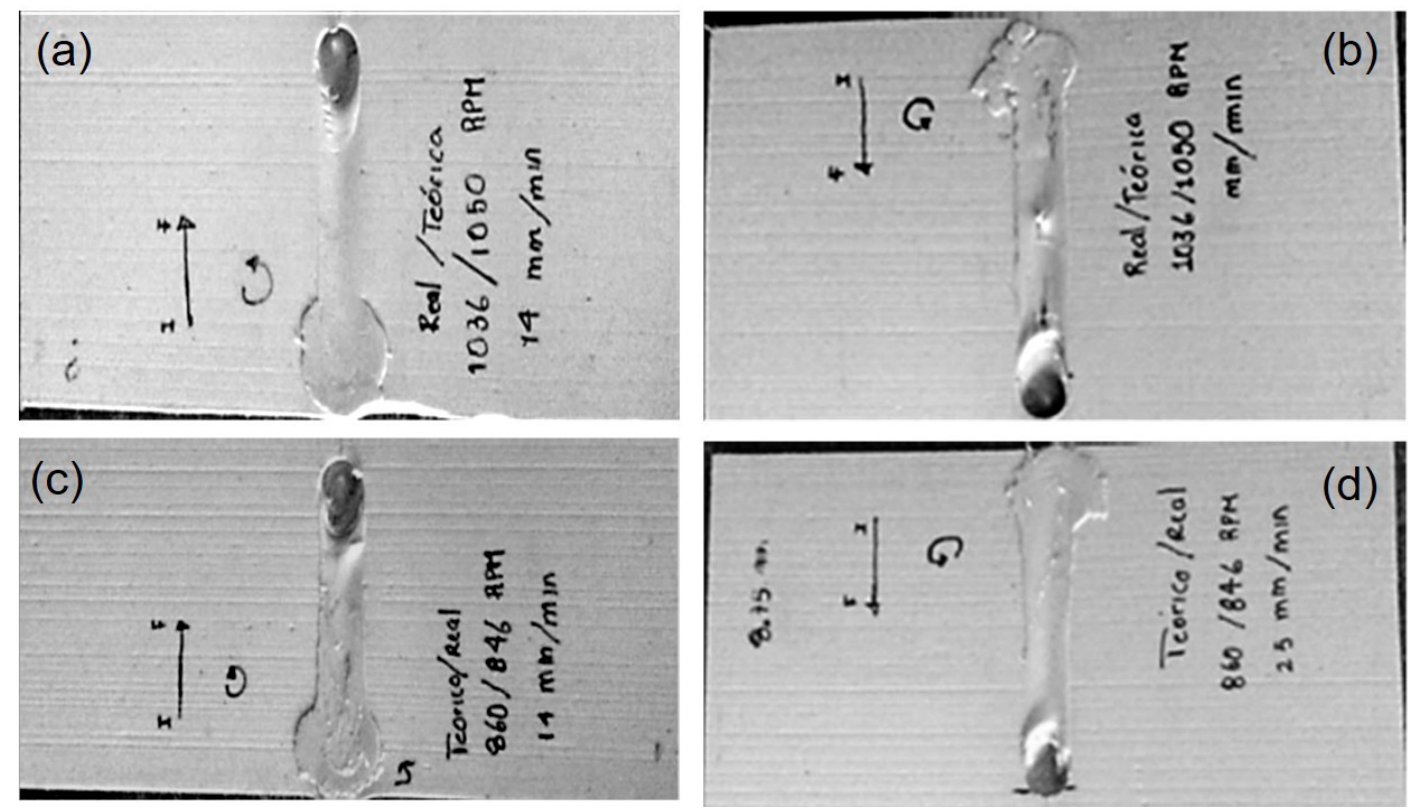

Figura 8. Soldaduras preliminares con herramienta no convencional. (a) $1036 \mathrm{rpm} / 14 \mathrm{~mm} \cdot \mathrm{min}^{-1}$; (b) $1036 \mathrm{rpm} / 25 \mathrm{~mm} \cdot \mathrm{min}^{-1}$; (c) $846 \mathrm{rpm} / 14 \mathrm{~mm} \cdot \mathrm{min}^{-1}$; (d) $846 \mathrm{rpm} / 25 \mathrm{~mm} \cdot \mathrm{min}^{-1}$. 
Las soldaduras obtenidas con la herramienta no convencional cumplieron con los criterios de selección establecidos, gracias al diseño de la herramienta con hombro no rotacional lo que permite la disminución del calor friccional, como lo establece Rezgui et al. [8]. Además, se encontró que los parámetros adecuados en la ventana de procesos, para realizar las soldaduras finales con la herramienta no convencional están entre 1036 y 847 rpm para velocidad de rotación y entre 14 y $25 \mathrm{~mm} \cdot \mathrm{min}^{-1}$ para velocidad de avance, usando un tiempo de permanencia (dwell time) de 5 segundos. Debido a los resultados anteriores, se ha decidido en este trabajo evaluar solo las soldaduras realizadas con la herramienta no convencional con el fin de evaluar la soldabilidad del PEAD desde el punto de vista aplicativo.

\subsection{Soldaduras finales}

Se realizaron soldaduras para evaluaciones finales con la herramienta no convencional dentro de la ventana de procesos que se muestra en la Tabla 1. Las juntas soldadas con estos parámetros tuvieron una longitud de $122 \mathrm{~mm}$, usando rotación del pin en sentido anti-horario. La ventana de procesos es una réplica de los resultados de las soldaduras preliminares y los resultados se muestran en la Figura 9.

En las juntas soldadas con alta velocidad de rotación se observó un pequeño tramo del cordón con espesor bajo, principalmente hacia el final del mismo. Es probable que esto haya sido ocasionado por el mayor calor friccional que se genera a mayores velocidades de rotación. A pesar de estas observaciones, anteriormente mencionadas, las juntas soldadas poseen apariencia física y rugosidad aceptables y no se observan discontinuidades en forma de vacíos en la superficie. Los valores de la relación $\mathrm{R}$, dados por la Ecuación 1 , se muestran en la Tabla 2. La relación más baja de R muestra la mejor apariencia superficial del cordón, como se observa en la Figura 9.

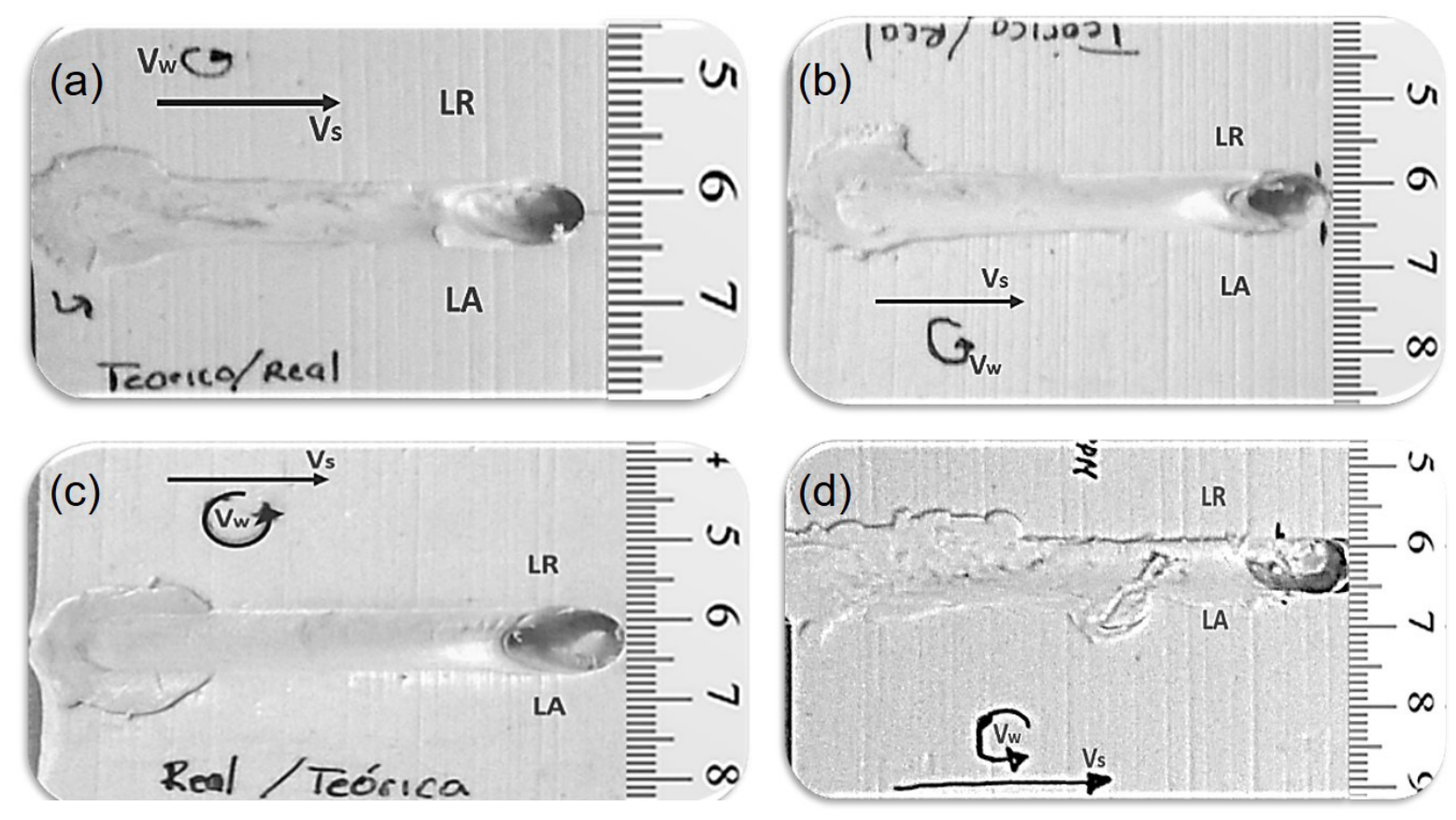

Figura 9. Soldaduras para evaluación final con herramienta no convencional. (a) $846 \mathrm{rpm} / 14 \mathrm{~mm} . \mathrm{min}^{-1}$; (b) $846 \mathrm{rpm} / 25 \mathrm{~mm} \cdot \mathrm{min}^{-1}$; (c) $1036 \mathrm{rpm} / 14 \mathrm{~mm} \cdot \mathrm{min}^{-1}$; (d) $1036 \mathrm{rpm} / 25 \mathrm{~mm} \cdot \mathrm{min}^{-1}$. LA: Lado de avance; LR: Lado de retroceso; Vw: velocidad de rotación; Vs: velocidad de avance.

Tabla 2. Valores de R para los parámetros evaluados experimentalmente.

\begin{tabular}{ccc}
\hline Velocidad de rotación $(\mathbf{r p m})$ & Velocidad de avance $\left(\mathbf{m m} \cdot \mathbf{m i n}^{-\mathbf{1}}\right)$ & $\mathbf{R}\left(\mathbf{r e v} \cdot \mathbf{m m}^{-1}\right)$ \\
$\mathbf{1 0 3 6}$ & 25 & 0.0241 \\
$\mathbf{1 0 3 6}$ & 14 & 0.0135 \\
$\mathbf{8 4 6}$ & 25 & 0.0295 \\
$\mathbf{8 4 6}$ & 14 & 0.0165 \\
\hline
\end{tabular}




\subsection{Caracterización de juntas soldadas}

\subsubsection{Observación macroscópica}

La exploración macroscópica de las juntas soldadas determinó la existencia de tres zonas distinguibles en la sección transversal, estas son: la zona del material base (MB), la zona agitada (ZA) y la línea de interfase (I), como se observa en la Figura 10, donde la línea de interfase fue remarcada de manera digital para hacerla distinguible. A partir del análisis macroestructural, se evidenció que las soldaduras obtenidas no presentaron discontinuidades tales como, vacíos, porosidades, falta de calentamiento, grietas u otros, en el interior del cordón que pudieran transgredir la integridad de la junta. Se observó que, al aumentar la velocidad de avance es notorio el exceso de material que se produce como sobremonta en la junta, además, aumenta el ancho de la zona agitada.
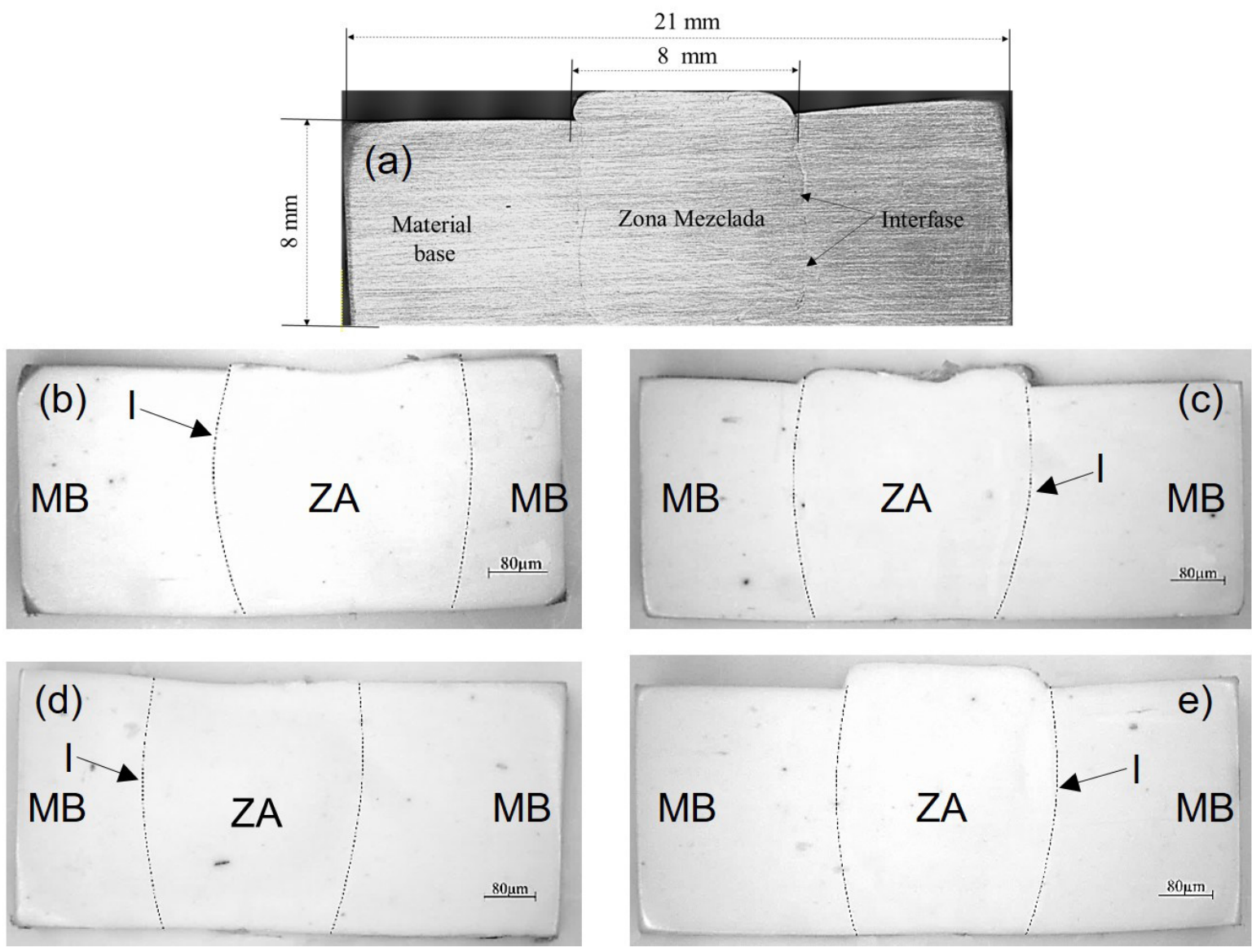

Figura 10. Sección transversal mostrando la apariencia de las juntas soldadas. (a) Imagen con microscopio confocal; (b) $1036 \mathrm{rpm} / 14 \mathrm{~mm} \cdot \mathrm{min}^{-1}$; (c) $1036 \mathrm{rpm} / 25 \mathrm{~mm} \cdot \mathrm{min}^{-1}$; (d) $846 \mathrm{rpm} / 14 \mathrm{~mm} \cdot \mathrm{min}^{-1}$; (e) $846 \mathrm{rpm} / 25 \mathrm{~mm} \cdot \mathrm{min}^{-1}$. MB: material base; ZA: zona agitada; I: Interfase.

Por otro lado, cuando la velocidad de avance disminuye la línea de interfase es menos notoria debido al calentamiento más homogéneo. Esto puede explicarse porque se genera más calor friccional debido a que las revoluciones por minuto en un mismo lugar son mayores y, por el contrario, cuando la velocidad de avance es mayor, el material ablandado, el cual fluye según el giro del pin roscado, tiene menos tiempo para lograr cohesión homogénea entre las distintas zonas de la junta generando una línea de interfase más notoria [9].

\subsubsection{Durezas}

Los resultados de los barridos de durezas se muestran en la Figura 11. En general las durezas son menores en la zona agitada con respecto al material base. Lo anterior es explicado porque los polímeros alteran sus propiedades en relación al recalentamiento. Los parámetros usados en la ejecución de las soldaduras por fricción agitación 

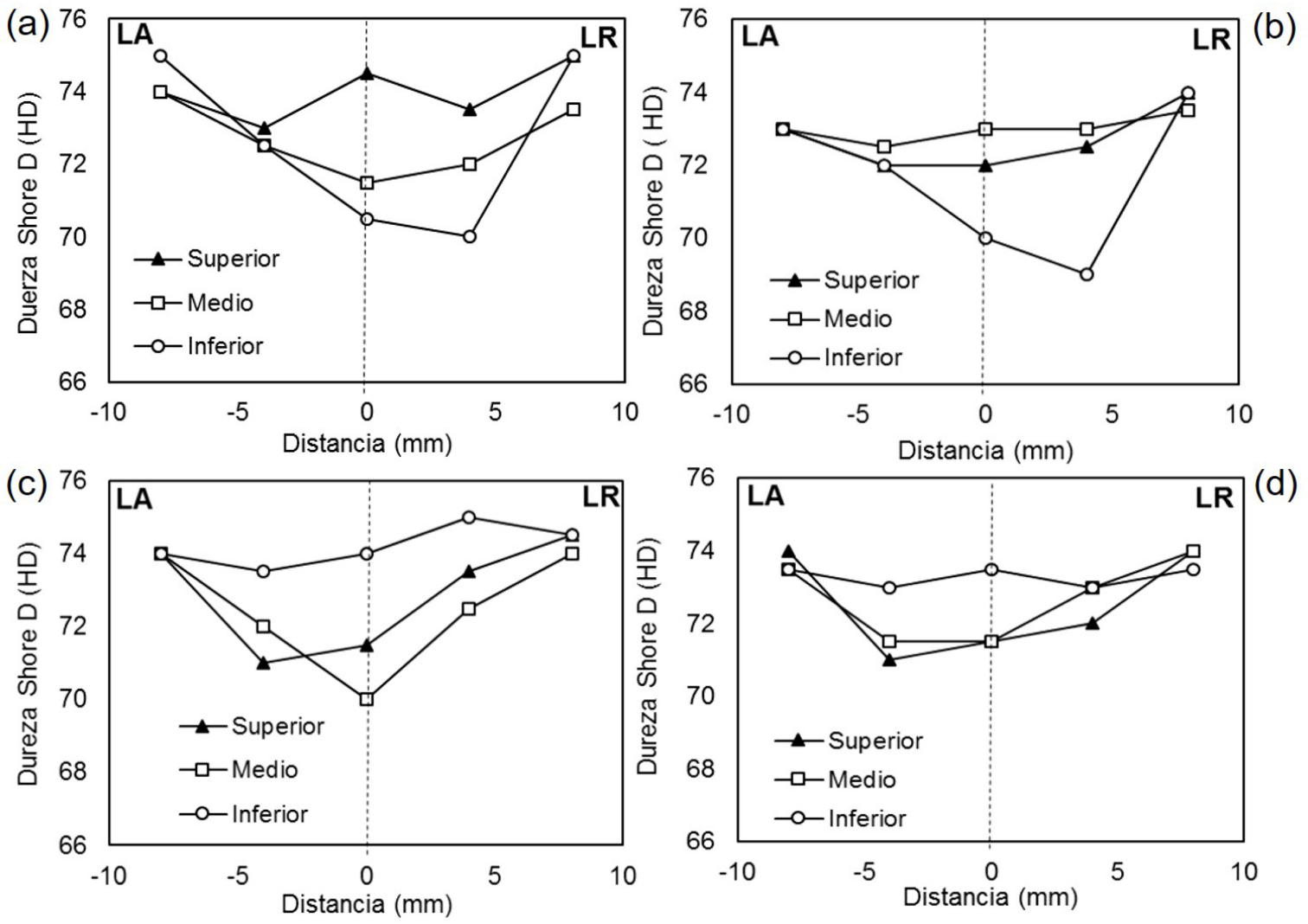

Figura 11. Resultados de los barridos de durezas. (a) $1036 \mathrm{rpm} / 14 \mathrm{~mm} \cdot \mathrm{min}^{-1}$; (b) $1036 \mathrm{rpm} / 25 \mathrm{~mm} . \mathrm{min}^{-1}$; (c) $846 \mathrm{rpm} / 14 \mathrm{~mm} \cdot \mathrm{min}^{-1}$; (d) $846 \mathrm{rpm} / 25 \mathrm{~mm} \cdot \mathrm{min}^{-1}$. LA: Lado de avance; LR: Lado de retroceso.

definitivas efectuaron ligeros cambios en la dureza del polietileno de alta densidad. De acuerdo con lo observado en los gráficos de durezas, es perceptible que a mayores velocidades de rotación y de avance, las durezas dentro de la zona agitada son menores. La velocidad de rotación afecta la distribución de durezas, con mayor influencia en el lado de retroceso a medida que la velocidad de rotación crece. Y caso contrario, observándose una leve acentuación del lado de avance con velocidades de rotación menores.

En la Figura 12 se muestra el comportamiento del valor medio de las durezas al interior de la zona agitada (los primeros $4 \mathrm{~mm}$ con respecto al centro de soldadura), con relación al lado de retroceso, al lado de avance y al centro de la soldadura. Los gráficos de barras muestran que la dureza en las juntas soldadas aumenta hacia el lado de retroceso (LR) a medida que la velocidad de rotación baja. Por otro lado, los cambios en el valor de dureza hacia el lado de avance son casi imperceptibles para cualquier velocidad de rotación y de avance, manteniéndose siempre por debajo del valor del material base. Así mismo, se observa que el valor medio de la dureza en la zona agitada, independiente de su ubicación, es siempre menor que la del material base (línea punteada). En general la dureza media de las juntas soldadas disminuye con el aumento de las velocidades de rotación y de avance [6].

En la Figura 13 se muestra la vista superior de la junta soldada, con los parámetros $1036 \mathrm{rpm}$ y $25 \mathrm{~mm} \cdot \mathrm{min}^{-1}$, donde es posible observar diferencias en el flujo de material dentro de la zona agitada tanto del lado de avance $\left(\mathrm{I}_{\mathrm{LA}}\right)$ como del lado de retroceso $\left(\mathrm{I}_{\mathrm{LR}}\right)$. Se observa que el flujo en el lado de avance presenta marcados cambios de dirección, partiendo suavemente desde la interfase, siguiendo la rotación de la herramienta. El lado de retroceso presenta signos de un flujo más estable, el cual, parece confinar al mismo desde la zona agitada hacia la línea de la junta soldada, mostrando una separación fuertemente marcada entre la interfase y el material base. El comportamiento anterior puede ser explicado por la mayor temperatura generada en el lado de avance (material que es arrancado al entrar en contacto con la herramienta y es arrastrado por el sentido de giro), mientras el lado de retroceso es más frío, proceso que es más acentuado a medida que la velocidad de rotación disminuye [10]. 
Soldabilidad de Polietileno de Alta Densidad Usando Soldadura por Fricción-agitación con una Herramienta de Hombro no Rotacional

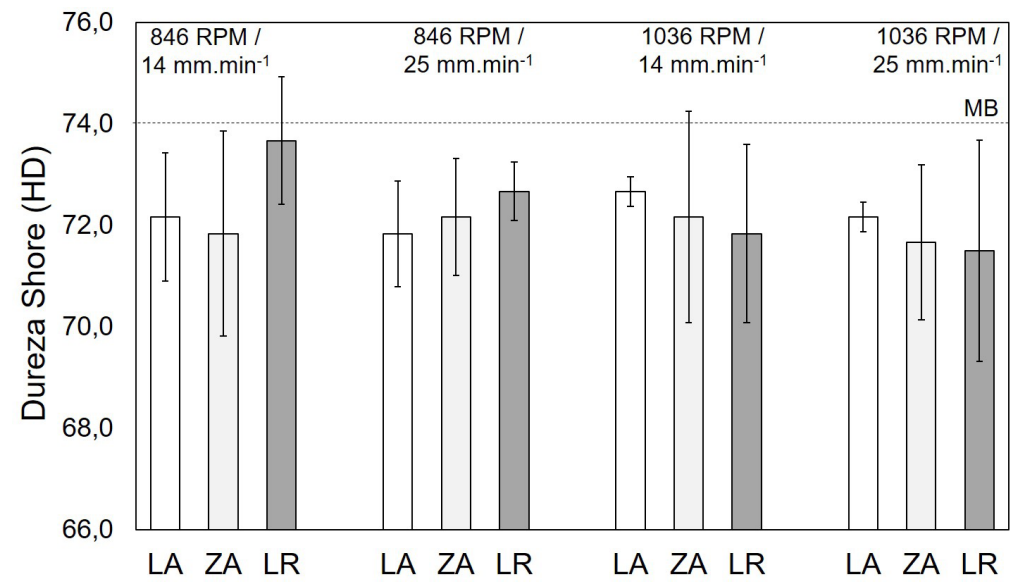

Figura 12. Valor medio de durezas al interior de la zona agitada, mostrando el comportamiento en: Lado de avance LA; Lado de Retroceso (LR), zona del centro (ZA), con relación a la interfase. MB: Material base.

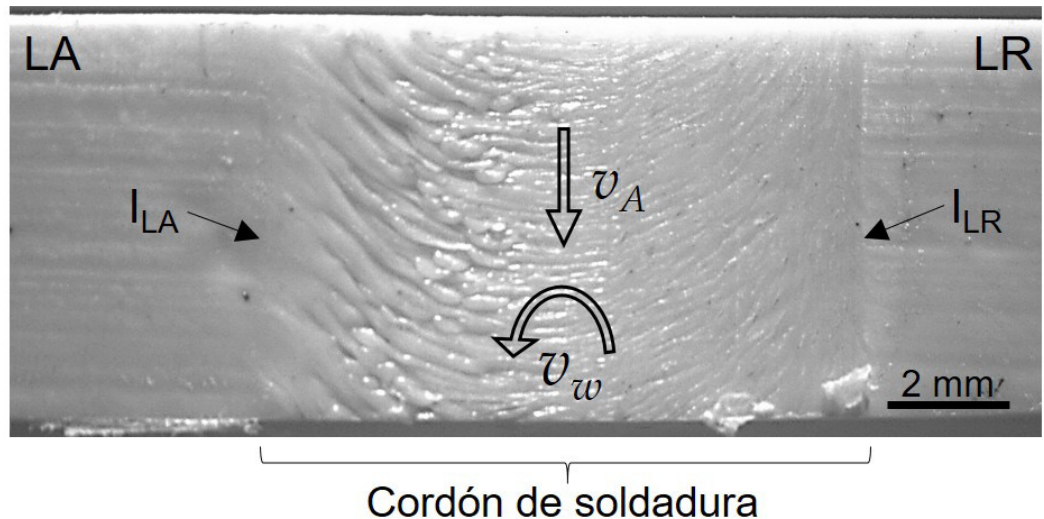

Figura 13. Detalle de la junta soldada en PEAD desde una vista superior. LA: Lado de avance; LR: Lado de retroceso; ILA: Interface en lado de avance; ILR: Interface en lado de retroceso.

El fenómeno de la diferenciación de flujos observados en la zona agitada puede influir sobre las durezas en cada zona de la región agitada, dependiendo de la velocidad de rotación. La diferencia de temperaturas y régimen de flujo del material en ambos lados de la zona agitada, podría explicar porque el lado de avance disminuye la dureza de manera más drástica en comparación al lado de retroceso, como fue observado en la Figura 12. De manera similar, este comportamiento puede explicar lo observado en otros trabajos similares que han estudiado el comportamiento a tracción de este tipo de juntas soldadas y presentan rotura en el lado de retroceso, mostrando comportamiento frágil al usar parámetros similares a los usados en este trabajo [7,8,12-14]. Debido a las altas velocidades propias del proceso de FSW, es posible que al polímero no le dé tiempo para relajar los esfuerzos al solidificar, dando como resultado desviaciones dimensionales o irregularidades en la zona agitada y cambios en sus propiedades mecánicas. Además de que se pierde la orientación de las cadenas de PEAD que se produce por el proceso de extrusión de la lámina.

\section{Conclusiones}

El estudio de la soldabilidad de polietileno de alta densidad (PEAD) ha confirmado la posibilidad de obtener juntas soldadas por el proceso de soldadura por fricción-agitación, usando una máquina fresadora universal y dos tipos de herramientas, una convencional y otra con hombro no rotacional, denominada no convencional. Los resultados mostraron que la herramienta no convencional, al poseer un hombro no rotacional, produce soldaduras continúas y con pocos defectos visuales en comparación a las soldaduras obtenidas con la herramienta 
convencional de hombro giratorio. Estas últimas juntas soldadas presentaron diversas discontinuidades dentro del cordón bajo parámetros similares. El bajo aporte de calor suministrado por la herramienta no convencional, como consecuencia de la fijación del movimiento rotacional del hombro, es la más probable causa de la reducción de discontinuidades en las soldaduras de PEAD. De acuerdo con la exploración macroscópica y microscópica de las juntas soldadas, mostraron ausencias de vacíos y juntas completamente consolidadas con la herramienta no convencional. La dureza no presentó grandes variaciones en las regiones de soldadura, salvo dentro del cordón donde se presentó una leve diferencia en la interfase de los lados de avance y retroceso. La diferencia fue evidenciada por microscopia mostrando flujo de material específico, mostrándose más agitado del lado de avance y menos agitado del lado de retroceso.

\section{Agradecimientos}

Los autores agradecen a la Universidad Autónoma del Caribe por el apoyo económico suministrado a través del proyecto CONV-I-004-P012. También agradecen la inmensa y desinteresada colaboración del Dr. Juan F. Santa del Instituto Tecnológico Metropolitano (ITM) y del profesor Ricardo Mendoza de la Universidad Autónoma del Caribe (UAC).

\section{Referencias}

[1] Nandan R, Debroy T, Bhadeshia HKDH. Recent advances in friction-stir welding-process, weldment structure and properties. Progress in Materials Science. 2008;53(6):980-1023. http:// dx.doi.org/10.1016/j.pmatsci.2008.05.001.

[2] The Welding Institute. "Friction stir welding" [online]. Cambridge; 2016 [acceso en 2016 ago 19]. Disponible: http://www.twi-global. com/capabilities/joining-technologies/friction-processes/ friction-stir-welding/.

[3] Pirizadeh M, Azdast T, Rash Ahmadi S, Mamaghani Shishavan S, Bagheri A. Friction stir welding of thermoplastics using a newly designed tool. Materials \& Design, Netherlands. 2014;54:342347. http://dx.doi.org/10.1016/j.matdes.2013.08.053.

[4] Throughton M. Friction stir welding. In: Andrew W. Handbook of plastics joining. 2. ed. Norwich: William Andrew Inc; 2008. $600 \mathrm{p}$.

[5] Thomas WM, Nicholas ED, Needhan JC, Murch MG. International patent application PCT/GB92/02203 and GB patent application 9125978.8. London UK Patent Office, 1991. v. 6.

[6] Panneerselvam K, Lenin K. Joining of Nylon 6 plate by friction stir welding process using threaded pin profile. Materials \& Design. 2014;53:302-307. http://dx.doi.org/10.1016/j. matdes.2013.07.017.

[7] Kiss Z, Czigany T. Microscopic analysis of the morphology of seams in friction stir welded polypropylene. Express Polymer Letters. 2012;6(1):54-62. http://dx.doi.org/10.3144/ expresspolymlett.2012.6.

[8] Rezgui MA, Ayadi M, Cherouat A, Hamrouni K, Zghal A, Bejaoui S. Application of Taguchi approach to optimize friction stir welding parameters of polyethylene. In: ICEM 14th International Conference on Experimental Mechanics - EPJ Web of Conferences 6; 2010 July 4-9; Poitiers, France. EDP Sciences, 2010. p. 8. https://doi.org/10.1051/epjconf/20100607003.

[9] Inaniwa S, Kurabe Y, Miyashita Y, Hori H. Application of friction stir welding for several plastic materials. In: Proceedings of the 1st International Joint Symposium on Joining and Welding: 2013 November 6-8; Osaka, Japan. Amsterdam: Woodhead Publishing; 2014. p. 137.

[10] Mishra RS, Ma ZY. Friction stir welding and processing. Materials Science and Engineering R Reports. 2005;50(1):1-78. http:// dx.doi.org/10.1016/j.mser.2005.07.001.

[11] American Society for Testing and Materials. ASTM D2240-05 standard test method for rubber property: durometer hardness. West Conshohocken: ASTM; 2010. p. 13.

[12] Arici A, Sinmazçelýk T. Effects of double passes of the tool on friction stir welding of polyethylene. Journal of Materials Science. 2005;40(12):3313-3316. http://dx.doi.org/10.1007/ s10853-005-2709-x.

[13] Aydin M. Effects of welding parameters and pre-heating on the friction stir welding of UHMW-polyethylene. Polymer-Plastics Technology and Engineering. 2010;49(6):595-601. http://dx.doi. org/10.1080/03602551003664503.

[14] Oliveira PHF. Estudo de viabilidade da soldagem de termoplásticos por" Friction Spot Welding"(FSpW). Soldagem e Inspeção. 2012;17:93-103. 